and circadian rhythms, is closely related to 5 - $\mathrm{HT}$, it seems interesting to study the role of melatonin in FS.

Objectives The aim of the study was to examine the total 24-hr 5 -HT and melatonin secretion in patients with FS and chronic low back pain (LBP) in relation to pain intensity, symptoms of anxiety/depression, and the severity of sleep disturbance.

Methods The 24-hr urinary excretion of the 6-sulphatoxymelatonin (aMT6s, main catabolite of melatonin) and the 5-hydroxyindoleacetic acid (5-HIAA, main catabolite of 5-HT) were measured using ELISA in women with FS $(n=18)$ and women with LBP $(n=15)$. The groups were matched with regard to age, diet, and the intensity of pain. Clinical measures of pain intensity, no of tender points, pain threshold (dolorimetry), psychological profile (SCL-90-R), and the severity of sleep disturbance (Schlaffragebogen-A and -B, SF-A and SF-B) were evaluated.

Results The excretion of aMT6s and 5-HIAA was lower in FS when compared to LBP $(8.79 \pm 1.90$ micrograms $/ 24$ hrs vs $12.53 \pm 3.01 \mathrm{micrograms} / 24 \mathrm{hrs}$ and $4.72 \pm 0.36 \mathrm{mg} / 24 \mathrm{hrs}$ vs $8.79 \pm 1.47 \mathrm{mg} / 24 \mathrm{hrs}$, respectively), but the difference was significant with regard to 5-HIAA only ( $<<0.03$ ). No correlation was found between the aMT6s and 5-HIAA within the study grups. In both groups, there was no relationship between the clinical measures of pain and tenderness and the excretion of the two catabolites. In FS, aMT6s excretion correlated weakly with the sleep quality (SQ, SF-B) $(\mathrm{r}=0.4966, \mathrm{p}<0.05)$ and the symptom duration $(\mathrm{r}=-0.5101, \mathrm{p}<0.05)$. When compared with the LBP group, the FS patients scored significantly higher on the G1 and G4 (somatization and depressivity) scales of SCL90-R, ( $\mathrm{p}<0.05$ for both comparisons) and the FRS subscale (feeling of recovery after sleep) of SF-A and SF-B ( $p<0.05$ and $\mathrm{p}<0.001$, respectively).

Conclusion Since the 24-hr urinary excretion of aMT6s closely follows the total 24-hr melatonin secretion, our results do not support the hypothesis of the disturbed metabolism of melatonin in FS. The small differences seen may result from the differences in the severity of mood and sleep disturbance. Further research is necessary to look at possible abnormalities in the circadian rhythm of melatonin secretion in FS.

\section{SAT0145 PERSONALITY STRUCTURE IN PATIENTS WITH PRIMARY FIBROMYALGIA AND REUMATOID ARTHRITIS}

${ }^{1} \mathrm{G}$ Vezyroglou, ${ }^{1} \mathrm{M}$ Myriokefalitakis, ${ }^{1} \mathrm{G}$ Papadimitriou, ${ }^{1} \mathrm{~A}$ Kotrotsios, ${ }^{1} \mathrm{C}$ Antoniadis, ${ }^{2} \mathrm{~K}$ Michelidakis. ${ }^{1}$ Psychiatry and Rheumatlogy; ${ }^{2}$ Rheumatology, Asklipieion Hospital Voula, Athens, Greece

\subsection{6/annrheumdis-2001.604}

Background Psychiatric symptoms occur in patients with chronic pain. It is well known that patients with rheumatoid arthritis present symptoms of depression to various degrees of intensity. Primary fibromyalgia syndrome is a form of non articular rheumatism characterised by generalised aches, pains, tender points, stiffness, and fatigue. There is an interest association between depression and pain.

Objectives The present study examines the personality structure of the patients with primary fibromyalgia syndrome and rheumatoid arthritis.

Methods Fifty (50) patients with rheumatoid arthritis and twenty-five (25) with fibromyalgia combared with fifty (50) nonpatients without pain. All patients presented the basic criteria and symptoms for rheumatoid arthritis (based in diagnostic criteria of the ARA revised at 1987 and the ACR 1990 diagnostic criteria for the fibromyalgia). The psychometric instruments are; a) HDHQ [Hostility and Direction of Hostility]. b) The SCL90R [Symptom Check List-90R]. c) DSSI-SAD[Delusions Symptoms Inventory-State of Anxiety and Depression].

Results The patients with primary fibromyalgia presents higher scores of depression and somatization than patients with rheumatoid arthritis.

Conclusion Probably the patients with rheumatoid arthritis are reasonable and accepted from this parents because is evident in our disability.

\section{REFERENCES}

1 Moutsopoulos H, Chused TM, Mann D, Clippell JH, et al. Sjogren's syndrome. Ann Int Med. 1980;92:212

2 Krol B, Sanderman R, Suurmeijer TP. Social support and quality of life concepts measurement and research. Patient Educ Couns. 1993;20:101-20

\section{SAT0146 RESULTS OF FOUR-YEARS EXPERIENCE IN FIBROMYALGIA FOLLOW-UP OUTPATIENT CLINIC}

Y Gursel, S Ergin, B Corekci. Physical Medicine and Rehabilitation, University of Ankara, Faculty of Medicine, Ankara, Turkey

\subsection{6/annrheumdis-2001.605}

Background Fibromyalgia, well-defined disease with widespread pain and tender points is still a challenge. Since it?s medically unexplained, the therapy remains empiric. So we need to assess our therapy results in longterm.

Objectives To determine the demographic, clinical properties with therapy results of patients followed in the Fibromyalgia Out-patient Follow-up Clinic (FOFC).

Methods In this study the records of the patients registered to FOFC were searched retrospectively. Records included demographic data, existing medical problems, symptoms related to fibromyalgia, VAS and Likert scale (0-4) for pain, scale scoring sleep disorder, number of tender points (TP), Fibromyalgia Impact Questionnaire (FIQ), Health Assessment Questionnaire (HAQ), Beck Depression Scale (BDS), laboratory assessments and therapy given. Patients were assessed by one trained researcher and by either one of the supervisors (YG, SE) at each visit. One hundred-twentythree records out of 166 were found to be eligible for analysis with sufficient data. SPSS 9.0 was used for statistical analysis.

Results Twenty-four patients $(19,5 \%)(\mathrm{M}=3, \mathrm{~F}=21)$ out of 123 were excluded with other diagnosis after initial assessment or after a few visits. Of these patients $95 \%$ had fatigue, $91 \%$ headache, $75 \%$ widespread pain, and 50\% sleep disorder. Ninety-nine patients diagnosed as fibromyalgia were all female with mean age of 40,89 years, mean duration of symptoms 56,90 months. Eighty-one were married, 8 single and 9 widow, 21 had a job and 78 were housewives. The most common symptom was fatigue (99\%) followed by widespread pain (91\%), headache $(84 \%)$, and sleep disorders (79\%). Initial mean values for VAS was $72,26 \mathrm{~mm}$, Likert scale was 2,71, number of TP was 14,02 , HAQ was 8,74 and BDS was 16,64 . VAS showed good correlation with Likert scale $(\mathrm{r}=, 619)$, 6th and 9th items of FIQ ( $r=, 453, r=, 418$ ) but weak correlation with sleep scores, HAQ, interestingly no correlation with TP. Tender points did not show significant correlation with any other of the items. Patients were treated with TCAs and SSRIs, NSAID and analgesics were added when needed, physical therapy modalities, ROM, stretching and aerobic exercises were used in some patients or combined to other therapies. Overall analysis showed 
significant improvement in VAS $(\mathrm{p}=, 032)$, sleep scores $(\mathrm{p}=$, $035)$, FIQ item rating fatigue ( $\mathrm{p}=, 002)$, FIQ items rating tension $(\mathrm{p}=, 028)$ and depression $(\mathrm{p}=, 004)$ but not in TP between initial and last visits. When records were analysed case by case, resistant patients were detected and only a few patients were seemed to have a longtime improvement, and some triggering factors such as emotional trauma, minor disease such as flu etc. found to affect patients? symptoms.

Conclusion Diagnosis, assessment and follow-up of patients with fibromyalgia is not easy because of subjective, variable symptoms. Most of the patients seemed to have good times and bad times. Results of the short term outcomes may be good, but most of the patients did not maintain improvement in the longer term, they also needed to change the drug because of loss of efficacy or side effects and other treatment modalities were combined.

\section{SAT0147 CORRELATION BETWEEN FIBROMYALGIA IMPACT QUESTIONNAIRE (FIQ) AND CLINICAL SYMPTOMS IN FIBROMYALGIA}

A Gür, M Karakoç, K Nas, R Çevik, AJ Saraç, F Erdogan. Physical Medicine and Rehabilitation, Dicle University, Diyarbakir, Turkey

\subsection{6/annrheumdis-2001.606}

Background Several well established instruments are available to measure health status and functional disability in persons with rheumatic diseases. Although both instruments are reliable and valid for several rheumatic disease groups, no formal psychometric testing of instruments have been reported for the population with fibromyalgia.

Objectives The study was designed to investigate the correlation between Fibromyalgia Impact Questionnaire (FIQ) and clinical symptoms, Health Assessment Questionnaire (HAQ) in patients with fibromyalgia in Turkey.

Methods FIQ is an assessment and evaluation instrument developed to measure physical functioning, work status, depression, anxiety, sleep, pain, stiffness, fatigue, and well being of fibromyalgia patients. We administered the FIQ and HAQ to 64 patients with fibromiyalgia and 48 healthy persons. Severity of relevant clinical symptoms (pain, fatigue, sleep disturbance, morning stiffness, muscle spasm, number of tender points, sensitivity of trigger points) were assessed.

Results The mean age of the patients and healthy group were $27,98+7,23$ and $28,75+5,67$, respectively $(p>0.05)$. Disease duration was 4,09+3,42 years. Correlation between FIQ and HAQ scores were rho $=0.46(p<0.01)$. Significant correlations were obtained between the FIQ scores and severity of clinical symptoms (morning stiffness $\mathrm{r}=0.42(\mathrm{p}<0.01$ ), fatigue $\mathrm{r}$ $=0.26(\mathrm{p}<0.05)$, pain $\mathrm{r}=0.67(\mathrm{p}<0.01)$, sensitivity $\mathrm{r}=$ $0.25(\mathrm{p}<0.05))$. In addition, significant correlations were obtained between the HAQ scores and number of tender points $r=0.28(p<0.05)$, morning stiffness $r=0.28(p<0.05)$, pain $\mathrm{r}=0.37(\mathrm{p}<0.01)$.

Conclusion In conclusion, the FIQ is a reliable and valid instrument for measuring functional disability in Turkish patients with fibromiyalgia. In addition, our study suggest that FIQ is more significant than HAQ in correlations with clinical symptoms in patients with fibromiyalgia.

\section{SAT0148 FIBROMYALGIA SYNDROME CHARACTERISED BY PATIENT-REPORTED SLEEP, FATIGUE, MOOD AND QUALITY OF LIFE}

${ }^{1} \mathrm{AM}$ Sesti, ${ }^{1} \mathrm{AE}$ Corbin, ${ }^{1} \mathrm{C}$ McLaughlin-Miley, ${ }^{1} \mathrm{M}$ Jaffe, ${ }^{1} \mathrm{~J}$ Moore, ${ }^{1} \mathrm{JP}$ Young, 'L LaMoreaux, ${ }^{1} \mathrm{U}$ Sharma, ${ }^{2} \mathrm{M}$ Versavel. ' Clinical, Pfizer, Ann Arbor, USA; ${ }^{2}$ Clinical, Pfizer, Fresnes, France

\subsection{6/annrheumdis-2001.607}

Background Fibromyalgia (FM) is a chronic condition of pain, tender points and multiple associated symptoms. A diagnosis is made by the ACR 1990 criteria requiring a history of widespread pain and pain in 11 of 18 tender points. Although pain is required for a diagnosis, the associated symptoms contribute to the syndromatic nature of the condition. This trial included measures of sleep, fatigue, mood, QoL and pain.

\section{Objectives}

Methods 529 fibromyalgia patients per ACR criteria were assessed at baseline after discontinuing meds used for FM pain/ insomnia (e.g. antidepressants, sedatives). The questionnaires were the Medical Outcomes Study Sleep Scale (MOS-SS), Multidimensional Assessment of Fatigue (MAF), Hospital Anxiety and Depression Scale (HADS) and SF-36. The MOS-SS has 12-items with an index score and 7 subscales: disturbance, snoring, awakening short of breath/headache (SOB/HA), adequacy, somnolence (range 0-100); quantity of sleep (reported hours); and, optimal sleep (\% with 7-8 h). Higher scores on the MOS-SS indicate more of the measured domain. The MAF has 16 items and 1 global index score from items measuring severity, distress, impact on activities and frequency of fatigue (range 1-50). The HADS has 14-items for anxiety/depression ranging from 0-21. Higher MAF and HADS scores indicate greater impairment. The SF-36 has 8 subscales measuring general health status with higher scores indicating better health states.

Results Demographics and pain scores are reported in the companion abstract by Corbin, et al. 2001. Baseline MOS-SS were: overall problems (62.3), disturbance (62.5), snoring (36.9), SOB/ HA (36.1), adequacy (19.7), somnolence (49.5), quantity (5.6), and $21.7 \%$ reported optimal sleep. The fatigue index was 38.9 and the HADS scores were mild with anxiety (10.1) and depression (8.6). SF-36 scores were physical function (40.5), role physical (15.2), bodily pain (27.6), general health (47.5), vitality (20.4), social functioning (48.8), role-emotional (46.0) and mental health (58.7).

Conclusion These data suggest that FM patients have impaired sleep and concomitant fatigue based on relatively high index scores. The predominant sleep problems were disrupted and inadequate sleep with a fair amount of somnolence. Patients reported low quality of life, particularly on domains of bodily pain, role physical, and vitality. Along with a high level of pain, impairments in sleep, fatigue and QoL were reported in this FM population.

\section{SAT0149 FIBROMYALGIA AND BENIGN JOINT HYPERMOBILITY SYNDROME}

P Borman, D Keskin, H Bodur. Department of Physical Medicine and Rehabilitation, Numune Education and Research Hospital, Ankara, Turkey

10.1136/annrheumdis-2001.608

Background Fibromyalgia syndrome is a chronic rheumatic condition characterised by widespread musculoskeletal pain. 